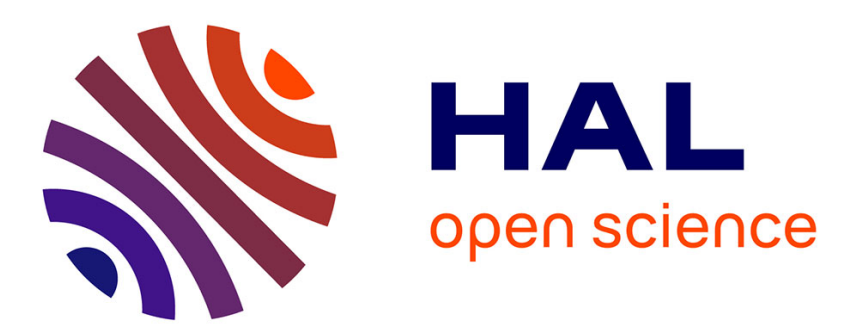

\title{
Measurement of the forbidden 6S - 8S M1 transition in caesium with an electric field
}

\author{
Ph. Jacquier, M. Broyer, L. Pottier, M.A. Bouchiat
}

\section{To cite this version:}

Ph. Jacquier, M. Broyer, L. Pottier, M.A. Bouchiat. Measurement of the forbidden $6 \mathrm{~S}-8 \mathrm{~S}$ M1 transition in caesium with an electric field. Journal de Physique, 1986, 47 (8), pp.1327-1334. 10.1051/jphys:019860047080132700 . jpa-00210325

\section{HAL Id: jpa-00210325 https://hal.science/jpa-00210325}

Submitted on 1 Jan 1986

HAL is a multi-disciplinary open access archive for the deposit and dissemination of scientific research documents, whether they are published or not. The documents may come from teaching and research institutions in France or abroad, or from public or private research centers.
L'archive ouverte pluridisciplinaire HAL, est destinée au dépôt et à la diffusion de documents scientifiques de niveau recherche, publiés ou non, émanant des établissements d'enseignement et de recherche français ou étrangers, des laboratoires publics ou privés. 
Classification

Physics Abstracts

$32.70 \mathrm{C}-32.90-31.90$

\title{
Measurement of the forbidden 6S - 8S $M_{1}$ transition in caesium with an electric field
}

\author{
Ph. Jacquier, M. Broyer (*), L. Pottier and M. A. Bouchiat \\ Laboratoire de Spectroscopie Hertzienne de l'E.N.S., associé au CNRS (U.A. 18), \\ 24, rue Lhomond, 75231 Paris Cedex 05, France
}

(Reçu le 31 janvier 1986, accepté le 18 avril 1986)

\begin{abstract}
Résumé. - La transition $6 \mathrm{~S}_{1 / 2}-8 \mathrm{~S}_{1 / 2}$ du césium est excitée, dans un champ électrique, avec un laser à colorant pulsé, de faible largeur spectrale, pompé par laser à azote. La détection se fait sur la lumière de fluorescence émise au cours de la désexcitation de $8 \mathrm{~S}_{1 / 2}$ vers $6 \mathrm{P}_{1 / 2}$. Nous obtenons le rapport des polarisabilités non diagonales scalaire et vectorielle : $\alpha / \beta=-4,8 \pm 0,5$. L'amplitude $M_{1}$ se manifeste par la polarisation électronique créée dans $8 S_{1 / 2}$ du fait de son interférence avec l'amplitude Stark induite. Nous obtenons $M_{1} / \beta=(-9,15 \pm 1,05) \mathrm{V} / \mathrm{cm}$. Grâce à une détermination théorique de $\alpha$, nous en déduisons $M_{1}=(-3,11 \pm 0,48) \cdot 10^{-5}\left|\mu_{\dot{B}}\right| / c$.
\end{abstract}

\begin{abstract}
The $6 \mathrm{~S}_{1 / 2}-8 \mathrm{~S}_{1 / 2}$ caesium transition is excited in a Stark field, with a pulsed narrow-band nitrogenpumped dye-laser. It is detected by monitoring the fluorescence light emitted in the $8 S_{1 / 2}-6 P_{1 / 2}$ decay. The scalarto-vector off-diagonal polarizability ratio is obtained : $\alpha / \beta=-4.8 \pm 0.5$. The $M_{1}$ amplitude is observed through the electronic polarization created in $8 \mathrm{~S}_{1 / 2}$ by its interference with the Stark-induced amplitude. We obtain $M_{1} / \beta=$ $(-9.15 \pm 1.05) \mathrm{V} / \mathrm{cm}$. Using a theoretical determination of $\alpha$, we deduce $M_{1}=(-3.11 \pm 0.48) \cdot 10^{-5}\left|\mu_{\mathrm{B}}\right| / c$.
\end{abstract}

\section{Introduction.}

The interest of highly forbidden $\mathbf{S}-\mathbf{S}$ transitions in heavy alkali elements for the search of parity violation $(\mathrm{PV})$ in atoms was pointed out long ago [1]. It is now well established since three experiments on the $6 \mathrm{~S}-7 \mathrm{~S}$ transition of caesium have been successfully performed [2-4].

Nonetheless, the study of these transitions remains of importance. The reason is that obtaining several consistent values of the same atomic quantity in different experimental conditions and through observation of different physical quantities will crucially reduce the possibility that significant sources of systematics may have been overlooked. Furthermore such studies provide new tests of the validity of the atomic calculations used in the prediction of the parity violation effect. This is why our group as well as others $[4,5]$ proceed further in studying these transitions.

(*) Present address : Laboratoire de Spectrométrie Ionique et Moléculaire, associé au CNRS (U.A. 171), 43, Bd du 11 Novembre 1918, 69622 Villeurbanne Cedex, France.
In this paper we study the $6 \mathrm{~S}-8 \mathrm{~S}$ transition of caesium. We have measured the scalar-to-vector polarizability ratio and the magnetic dipole amplitude of this transition $\left({ }^{1}\right)$. While Bouchiat et al. [2. 3. 7] used in the 6S - 7S transition a cw laser and lock-in detection, we have preferred a pulsed laser and box-car detection, to try out a new excitation and detection scheme which might improve the sensitivity of future PV measurements.

\section{Theory.}

1.1 THE $6 \mathrm{~S}_{1 / 2}-8 \mathrm{~S}_{1 / 2}$ TRANSITION : GENERAL FEATURES. - The relevant levels are shown in figure 1. The excited atoms are detected through the fluorescence light emitted in the $8 S-6 P_{1 / 2}$ decay $(761 \mathrm{~nm}$, branching ratio 0.223 ). This transition is preferred to $8 \mathrm{~S}-7 \mathrm{P}$, whose fluorescence $(\lambda \sim 4 \mu \mathrm{m})$ would be overshadowed by the thermal radiation of the hot $\left(20{ }^{\circ} \mathrm{C}\right)$ parts of the apparatus.

( ${ }^{1}$ ) This work is presented with more details in $\mathrm{Ph}$. Jacquier, Thèse de $3^{e}$ Cycle, Paris (1982), unpublished : reference [6]. 


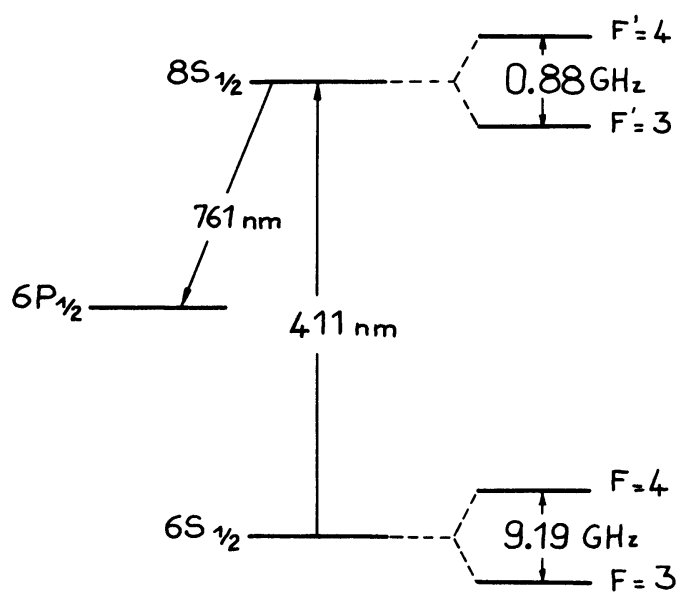

Fig. 1. - Energy levels of caesium involved in the present experiment (not to scale).

1.2 Principle of the Measurements : the Stark INTERFERENCE METHOD. - The basic idea for measuring such a tiny transition dipole amplitude as the $M_{1}$ amplitude is to let it interfere with a larger, known amplitude induced by an external dc electric field $\mathbf{E}$ [1]. This interference gives rise to an atomic orientation $\mathbf{P}$ which in turn results in circular polarization of the $8 \mathrm{~S}-6 \mathrm{P}_{1 / 2}$ fluorescence. The circular polarization ratio is exactly equal to the component of $\mathbf{P}$ in the direction of observation.

1.2.1 Effective transition dipole. - In the presence of an external dc field, the $6 \mathrm{~S}-8 \mathrm{~S}$ transition can be described by means of an effective transition dipole [1] :

$$
\begin{aligned}
& \left\langle 8 \mathrm{~S} m_{\mathrm{S}}^{\prime}|\mathbf{d}| 6 \mathrm{~S} m_{\mathrm{S}}\right\rangle= \\
& \quad=\left\langle m_{\mathrm{S}}^{\prime}\left|-\alpha \mathbf{E}-i \beta \sigma \times \mathbf{E}+M_{1} \sigma \times \hat{\mathbf{k}}\right| m_{\mathrm{S}}\right\rangle,
\end{aligned}
$$

where $\hat{\mathbf{k}}$ is the unit vector of the propagation direction of the laser beam and $\sigma$ is the Pauli operator of the electronic spin $\left({ }^{2}\right)$.

In this effective dipole, the first two terms are interpreted as the scalar and vector parts of the Starkinduced electric dipole. The theoretically predicted values of the scalar $(\alpha)$ and vector $(\beta)$ off-diagonal polarizability are respectively (in atomic units) [8] $\alpha=-306.3$ and $\beta=55.6$, whence

$$
E_{1}=-\alpha E=\left(1.63 \times 10^{-5}\left|\mu_{\mathrm{B}}\right| / c\right) \times(E \text { in } \mathrm{V} / \mathrm{cm})
$$

and

$$
\alpha / \beta=-5.5 \quad \text { (with about } 10 \% \text { uncertainty). }
$$

The third term is the magnetic dipole [1]. It originates entirely in relativistic many-body effects. Although its mechanism seems well understood [9] its theoretical

$\left({ }^{2}\right)$ A parity-violating contribution, such as considered in [7], is also expected, but is negligible in the present discussion. prediction from first-principle calculations, similar to those performed for $6 \mathrm{~S}-7 \mathrm{~S}$, is not expected to be accurate. On the other hand one can make some reasonable guess concerning the comparison of $M_{1}$ between different $6 \mathrm{~S}-n S$ transitions in Cs. Since $M_{1}$ results from exchange forces between $\mathrm{s}$ and $\mathrm{p}$ core electrons, the matrix element

$$
\left\langle 6 \mathrm{~S}_{1 / 2} 1 / 2\left|\mu_{z} / c\right| n \mathrm{~S}_{1 / 2} 1 / 2\right\rangle=M_{1}(6 \mathrm{~S} \rightarrow n \mathrm{~S})
$$

is expected to be proportional to the amplitudes of $6 \mathrm{~S}_{1 / 2}$ and $n \mathrm{~S}_{1 / 2}$ waves inside the core, i.e. to $\left(1 / v_{6}\right)^{3 / 2}\left(1 / v_{n}\right)^{3 / 2}$, where $v_{n}$ denotes the effective quantum number. We thus obtain :

$$
M_{1}\left(6 \mathrm{~S}_{1 / 2} \rightarrow 8 \mathrm{~S}_{1 / 2}\right) \sim 0.64 M_{1}\left(6 \mathrm{~S}_{1 / 2} \rightarrow 7 \mathrm{~S}_{1 / 2}\right),
$$

and from the empirical knowledge [10] of $M_{1}\left(6 \mathrm{~S}_{1 / 2} \rightarrow 7 \mathrm{~S}_{1 / 2}\right)$ :

$$
M_{1}\left(6 \mathrm{~S}_{1 / 2} \rightarrow 8 \mathrm{~S}_{1 / 2}\right) \sim-2.5 \times 10^{-5}\left|\mu_{\mathrm{B}}\right| / c .
$$

The contribution $M_{1 \mathrm{hf}}$ from hyperfine mixing between two $S$ states [1] does not contribute here since we are interested in $\Delta F=0$ components only.

From the above discussion we expect $M_{1}$ and $\alpha E$ amplitudes of comparable size in an applied field of a few $\mathrm{V} / \mathrm{cm}$. In our measurements the field is typically $100 \mathrm{~V} / \mathrm{cm}$, so the Stark-induced amplitudes are much larger than the $M_{1}$ amplitude.

1.2.2 Measuring $\alpha / \beta$. - From equation (1) the dependence of the fluorescence intensity on the electric field $\mathbf{E}$ and on the laser polarization $\varepsilon$ can be computed. Depending on which hyperfine component the laser is tuned to, the fluorescence intensity is :

$$
\left.\begin{array}{lrr}
4-4: & I=36 \alpha^{2}|\mathbf{E} \cdot \varepsilon|^{2}+15 \beta^{2}|\mathbf{E} \times \varepsilon|^{2}, \\
3-3: & I=28 \alpha^{2}|\mathbf{E} \cdot \varepsilon|^{2}+7 \beta^{2}|\mathbf{E} \times \varepsilon|^{2}, \\
3-4 \text { or } 4-3: I= & 21 \beta^{2}|\mathbf{E} \times \varepsilon|^{2} .
\end{array}\right\}
$$

If the laser does not resolve the hfs of the $8 \mathrm{~S}$ state at all, equations (4) should be rewritten as :

$$
\begin{aligned}
& 4-(3 \& 4): I=36 \alpha^{2}|\mathbf{E} \cdot \varepsilon|^{2}+36 \beta^{2}|\mathbf{E} \times \varepsilon|^{2}, \\
& 3-(3 \& 4): I=28 \alpha^{2}|\mathbf{E} \cdot \varepsilon|^{2}+28 \beta^{2}|\mathbf{E} \times \varepsilon|^{2} .
\end{aligned}
$$

Our real case approaches the conditions of equations (5) and (6).

In all cases the fluorescence intensity depends on the angle of the laser polarization with respect to the electric field. The comparison of the intensity obtained with $\varepsilon / / \mathbf{E}\left(\alpha^{2}\right.$ term alone) and $\varepsilon \perp \mathbf{E}\left(\beta^{2}\right.$ term alone) yields the ratio $\alpha^{2} / \beta^{2}$.

1.2.3 Electronic polarizations. - From equation (1) one can show that the electronic polarization $\mathbf{P}=$ $(2 / \hbar)\langle\mathbf{S}\rangle$ can be written as a sum of two contributions $\mathbf{P}=\mathbf{P}^{(1)}+\mathbf{P}^{(2)} \cdot \mathbf{P}^{(1)}$ is odd under reversal of the field $\mathbf{E}$, and depends on the linear polarization ratio of the laser beam. In contrast, $\mathbf{P}^{(2)}$ is even under reversal of $\mathbf{E}$, and depends on the circular polarization ratio. 
Thus $\mathbf{P}^{(1)}$ and $\mathbf{P}^{(2)}$ can easily be distinguished experimentally. But for terms of order $M_{1}^{2}$, neglected here throughout, $\mathbf{P}^{(1)}$ originates in interference of the $M_{1}$ term with either the $\alpha \mathbf{E}$ or $\beta \mathbf{E}$ term in equation (1), while $\mathbf{P}^{(2)}$ results from interference between the $\alpha \mathbf{E}$ and $\beta \mathbf{E}$ terms.

When the laser is tuned to the 4 - ( $3 \& 4$ ) transition and polarized linearly along $\mathbf{E}$, the $4-3$ transition does not contribute. (In Eq. (1) the scalar operator $-\alpha \mathbf{E} \cdot \boldsymbol{\varepsilon}$ has no matrix elements between states of different $F$, and $-i \beta \boldsymbol{\sigma} \times \mathbf{E} \cdot \boldsymbol{\varepsilon}$ vanishes for $\boldsymbol{\varepsilon} / / \mathbf{E}$ ). So $\mathbf{P}^{(1)}$ originates only in the $4-4$ transition, and is given by

$$
\begin{aligned}
& \mathbf{P}^{(1)}=\frac{5(1+\beta / 8 \alpha)}{6\left(1+5 \beta^{2} / 12 \alpha^{2}\right)} \cdot \frac{M_{1}}{\alpha|E|} \hat{\mathbf{E}} \times \hat{\mathbf{k}}, \\
& \text { for } M_{1}^{2} \ll|\alpha \mathrm{E}|^{2},
\end{aligned}
$$

where $\hat{\mathbf{E}}=\mathbf{E} /|\mathbf{E}|$.

Measuring $\mathbf{P}^{(1)}$ yields, in principle, the ratio $M_{1} / \alpha$. In practice, possible depolarization of the $8 \mathrm{~S}$ state by collisions, or of the fluorescence light by imperfections in the optics, has to be allowed for. This is done by measuring with the same apparatus the contribution $\mathbf{P}^{(2)}$ used as a standard. When the laser is tuned to the 4 - (3\&4) transition and polarized circularly, the 4 - 3 component, which has no polarization $\mathbf{P}^{(2)}$, only dilutes the $\mathbf{P}^{(2)}$ component of the 4 - 4 transition. Since its intensity is only $2 \%$ of the intensity of the latter and since overlapping is incomplete, the dilution effect $(\sim 1 \%)$ is negligible in view of the experimental uncertainty. Finally $\mathbf{P}^{(2)}$ is given by

$$
\begin{aligned}
& \mathbf{P}^{(2)}=-\frac{5}{6} \frac{1}{1+5 \beta^{2} / 12 \alpha^{2}} \cdot \frac{\beta}{\alpha} \xi \hat{\mathbf{k}}, \\
& \text { for } M_{1}^{2} \ll|\beta E|^{2}, \quad|\alpha E|^{2},
\end{aligned}
$$

where $\xi= \pm 1$ is the sign of the circular polarization.

Once $\beta / \alpha$ has been measured the value (8) is known and $P^{(2)}$ can be used as a polarization standard. In particular the ratio $P^{(1)} / P^{(2)}$ yields a direct measurement of $M_{1} / \beta$ :

$$
M_{1} / \beta=-(1+\beta / 8 \alpha) P^{(1)} / P^{(2)}(\xi=+1) .
$$

In principle, the circular polarization ratio of the $8 \mathrm{~S}-6 \mathrm{P}_{1 / 2}$ fluorescence light emitted in a direction $\hat{\mathbf{k}}_{\mathrm{f}}$ is equal to the component $\mathbf{P} \cdot \hat{\mathbf{k}}_{\mathrm{f}}$ of the electronic polarization in this direction. To determine the latter we measure the former. We pass the fluorescence light through an analyser made of a quarter-wave plate followed by a polarizer whose axis is set at $45^{\circ}$ to the axes of the plate. Switching the plate alternately to the $+45^{\circ}$ or to the $-45^{\circ}$ position, we measure two transmitted intensities $I_{+}$and $I_{-}$and compute the circular polarization ratio $\left(I_{+}-I_{-}\right) /\left(I_{+}+I_{-}\right)$.

In order to avoid contamination of the observed polarization $\mathbf{P}^{(1)}$ by the larger polarization $\mathbf{P}^{(2)}$ we take advantage of their specific properties :

i) $\mathbf{P}^{(2)}$ is along the laser direction $\hat{\mathbf{k}}$ while $\mathbf{P}^{(1)}$ is normal to it, along $\hat{\mathbf{E}} \times \hat{\mathbf{k}}$;

ii) $\mathbf{P}^{(2)}$ vanishes when the laser is polarized linearly;

iii) $\mathbf{P}^{(1)}$ is odd under reversal of $\mathbf{E}$ while $\mathbf{P}^{(2)}$ is even.

Consequently we choose the detection direction transverse to the beam and to the field $\mathbf{E}$ (Fig. 2a) and we polarize the laser linearly along $\mathbf{E}$. We measure a quantity odd under E-reversal :

$$
p_{1}=\frac{1}{2}\left[\frac{I_{+\uparrow}-I_{-\uparrow}}{I_{+\uparrow}+I_{-\uparrow}}-\frac{I_{+\downarrow}-I_{-\downarrow}}{I_{+\downarrow}+I_{-\downarrow}}\right] .
$$

(The arrows indicate an electric field $+\mathbf{E}$ or $-\mathbf{E}$ respectively). An additional precaution consists in periodically applying a magnetic field to take advantage of the Hanle effect experienced by $\mathbf{P}^{(1)}$ in order to still better discriminate it. In a magnetic field $\mathbf{H}$ parallel to $\mathbf{E}$ (Fig. 2b) the contribution of $\mathbf{P}^{(1)}$ to the polarization signal is :

$$
p_{1}(H)=\frac{p_{1}(H=0)}{1+(H / \Delta H)^{2}}
$$

where $\Delta H$ is the Hanle width of the $8 \mathrm{~S}_{1 / 2}$ state ( $\sim 5$ gauss). We record the value of

$$
p_{1}^{*}=p_{1}(H=0)-p_{1}(H \gg \Delta H) .
$$

The quantity $p_{1}^{*}$ would coincide with $\mathbf{P}^{(1)} \cdot \hat{\mathbf{k}}_{\mathrm{f}}$ in the absence of depolarization. Although the experimental arrangement is not perfect, simultaneous use of the above mentioned criteria makes systematic errors arising from apparatus imperfections negligible [6].

In practice some polarization loss is expected, so that it is necessary to calibrate the measurement. For this we use as a standard polarization $\mathbf{P}^{(2)}$, whose value is known since we know the ratio $\alpha / \beta$ (Eq. (8)). $\mathbf{P}^{(2)}$, perpendicular to the observation direction, is not detected. In fact we can " turn " it at will, using Hanle effect in a magnetic field $\mathbf{H}$ parallel to $\mathbf{E}$ (Fig. 2b). But for the depolarization, the detected component

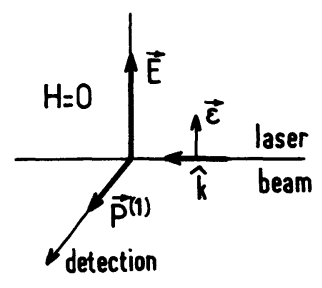

.a.

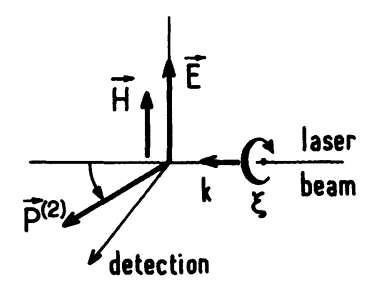

b.
Fig. 2. - Geometrical configurations : a) Observation of the electronic polarization component $\mathbf{P}^{(1)}$. No magnetic field; $\mathbf{E}:$ dc electric field; $\boldsymbol{\varepsilon}$ : linear polarization of the laser beam. b) Observation of component $\mathbf{P}^{(2)}$. $\mathbf{H}$ : Hanle magnetic field; $\xi= \pm 1$ : circular polarization of the laser beam. 
of $\mathbf{P}^{(2)}$ is then expected to be

$p_{2}=-\frac{5}{6}\left(1+5 \beta^{2} / 12 \alpha^{2}\right)^{-1} \frac{\beta}{\alpha} \frac{H / \Delta H}{1+(H / \Delta H)^{2}}\left(\xi \hat{\mathbf{k}} \times \hat{\mathbf{k}}_{\mathrm{f}} \cdot \hat{\mathbf{H}}\right)$

where $\Delta H>0$. Once again, for better discrimination we take advantage of the specific behaviour of $\mathbf{P}^{(2)}$ by measuring the E-even quantity :

$$
p_{2}(H)=\frac{1}{2}\left[\frac{I_{+\uparrow}-I_{-\uparrow}}{I_{+\uparrow}+I_{-\uparrow}}+\frac{I_{+\downarrow}-I_{-\downarrow}}{I_{+\downarrow}+I_{-\downarrow}}\right],
$$

for $H=\Delta H$ and for $H=0$. Finally we record the value of

$$
p_{2}^{*}=p_{2}(H=\Delta H)-p_{2}(H=0) .
$$

The corrections $p_{1}(H \gg \Delta H)$ and $p_{2}(H=0)$, expected to be zero, actually remain smaller than the noise. Moreover, the ratio of the observed to expected values of $p_{2}^{*}$ remains reasonable $(\sim 2 / 3)$.

The sign of $p_{1}^{*} / p_{2}^{*}$ is that of $\mathbf{P}^{(1)} / \mathbf{P}^{(2)}(\xi=+1)$ provided the state labeled $\uparrow$ in equations (11) and (14) corresponds to fields $\mathbf{E}$ and $\mathbf{H}$ of same direction (the sign of the circular analyser in the state labelled + disappears in the ratio $p_{1}^{*} / p_{2}^{*}$ ). The measurement then directly yields the sign of $M_{1} / \beta$ (Eq. (9)).

\section{Apparatus.}

A schematic diagram of the experimental set-up and of the data acquisition system is shown in figure 3 .

2.1 CELL; OVEN; COILS. - The caesium vapour is contained in a cell made of Corning 1720 glass, represented to scale in figure 4. Before assembling, the stainless steel electrodes are heated to $1000^{\circ} \mathrm{C}$ under hydrogen atmosphere, in order to eliminate most of the impurities that could otherwise react with the caesium. Diaphragms are attached to the bottom electrode to stop scattered laser light that would strike

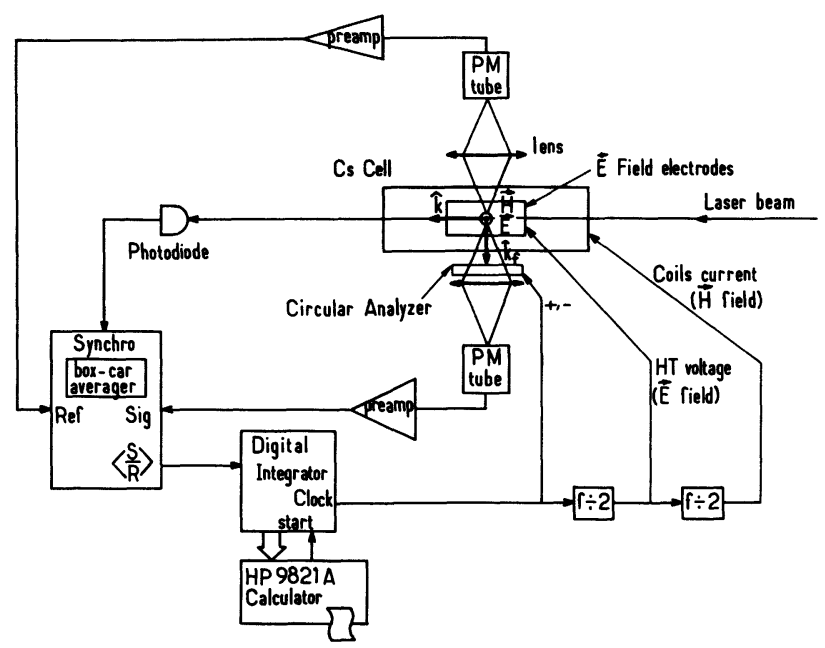

Fig. 3. - Scheme of the apparatus.

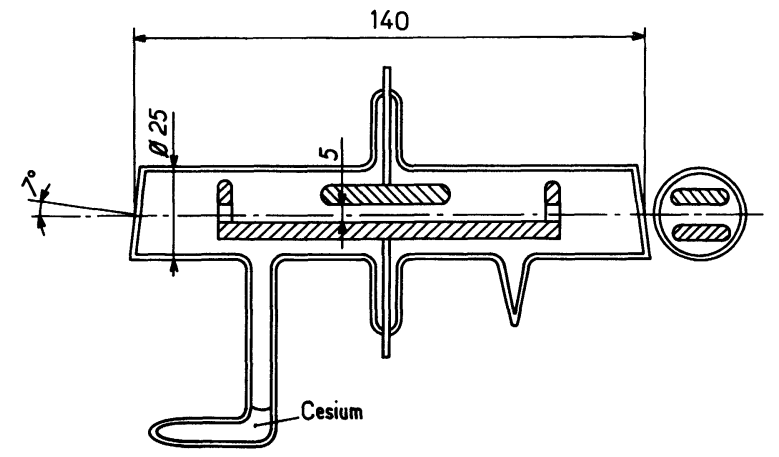

Fig. 4. - Corning 1720 caesium cell with stainless steel electrodes (side view and section view). The lower electrode is grounded and provided with two diaphragms.

the electrodes in the electric field region and give rise to photo-induced damaging discharges. After assembling, the cell is outgassed for $72 \mathrm{~h}$ at $400^{\circ} \mathrm{C}$, filled with a few grams of Cs and sealed. This cell is maintained in an oven at a temperature near $150^{\circ} \mathrm{C}$ for which the vapour pressure of Cs is roughly $8 \mathrm{mtorr}$. The cell with the oven (made of brass enclosed in insulating material) is placed in a pair of Helmholtz coils that provide the magnetic field (up to 20 gauss) together with a correction for the vertical component of the earth field. The effect of the horizontal component is made negligible by arranging the cell with its axis (direction of propagation of the laser beam) parallel to this component.

2.2 LASER. - The laser consists of a Molectron DL 300 used as an oscillator, followed by an amplifier. Both use a magnetically stirred dye cell with diphenylstilbene dye and are pumped transversely by the UV beam of a Molectron UV-1 000 nitrogen laser. $(10 \mathrm{~Hz}$ repetition rate; dye lifetime : $30 \mathrm{~min}$.)

The oscillator uses a 600 grooves $/ \mathrm{mm}$ grating in the seventh order with a telescope. Frequency selection is performed by an etalon inserted between the grating and the telescope. This etalon (Molectron DL 26, FSR $=15 \mathrm{GHz}$ ) consists of a silica disc coated on each face to give a finesse of roughly 20 . Since the refractive index of silica changes with temperature $\left(10^{-5} \mathrm{~K}^{-1}\right)$ the etalon has to be thermostatically controlled, otherwise the laser would sweep freely over our transition within a few minutes.

This oscillator stage delivers a few $\mu \mathrm{J}$ of laser light to the amplifier. The latter consists of a cell simply placed next to the output window of the oscillator and separated from it by a diaphragm. The delay of the pump beam on the amplifying cell was determined by trial and correction.

The resulting power output is roughly $90 \mu \mathrm{J}$. The linewidth, as deduced from observation of the transition, is $\sim 2 \mathrm{GHz}$ (see below). As mentioned in $\S 1$, the hfs of the $6 \mathrm{~S}_{1 / 2}$ level is fully resolved but that of the $8 \mathrm{~S}_{1 / 2}$ level is not at all. 
2.3 DeteCtor AND ANALYSER. - The fluorescence light is collected from the two sides of the cell by doublets of $f=100 \mathrm{~mm}, 80 \mathrm{~mm}$ diameter lenses and condensed onto the cathode of a C 31034 A photomultiplier from RCA. A multidielectric filter placed between the two lenses where the light is almost parallel selects the $8 \mathrm{~S}_{1 / 2}-6 \mathrm{P}_{1 / 2}$ fluorescence light, while an additional filter blocks the scattered light at $411 \mathrm{~nm}$.

On one side of the cell we detect the total fluorescence intensity. On the other side the light is passed through a circular analyser. The ratio of the latter channel to the former gives the polarization ratio and eliminates pulse-to-pulse fluctuations of the laser intensity or frequency. This provides the measurement of the quantity denoted by $I_{ \pm}$in equations (10) and (14). The circular analyser consists of a fixed Polaroid sheet following an approximately $5 \lambda / 4$ quartz plate stuck on a silica disc by molecular adhesion. The plate is mounted on a non-magnetic (bronze) ballbearing enabling a rotation of $\pm 90^{\circ}$, defined by adjustable mechanical stops. The rod that drives the rotating part is elastic, so as to maintain it pressed against the stop in either extreme position.

2.4 DAta ACQuisition. - Our data acquisition system consists of a Molectron LP 20 dual gated differential integrator (box-car), a home-made digital integrator and a HP 9821 A calculator. The box-car, triggered by the output of a photodiode placed in the laser beam, integrates the signals of the photomultipliers during $24 \mu \mathrm{s}$. Integration takes place again $300 \mu$ s later, after the signal of interest has gone to zero. The results are subtracted, eliminating the continuous background associated with the dark current of the photomultipliers (but the noise associated with this background is not eliminated).

The digital integrator is the heart of the system. For 4 seconds it reads the outputs of the box-car and integrates them digitally ("live time "). Then it commands the reversal of the analyser (or $\mathbf{E}$, or $\mathbf{H}$ ) and waits 1 second for the experiment to stabilize ( $\backsim$ dead time ") while transferring the results of the preceding live time to the calculator.

Since three parameters are reversed, $2^{3}=8$ successive live times are necessary to go through all possible sign combinations and complete one measurement cycle. The program thus stores eight numbers and reconstructs from them the polarization $p_{1}^{*}$ or $p_{2}^{*}$ according to formulae (12) and (15) of $\S 1$. The average and the standard deviation are then computed over many cycles.

\section{Experimental conditions.}

Table I summarizes typical experimental conditions. We have checked the proportionality of the fluorescence signal to the caesium number density and its linearity versus $E^{2}$. As shown in figure 5 , the signal observed off-resonance is approximately equal to the Stark induced signal for $E=20 \mathrm{~V} / \mathrm{cm}$. It originates

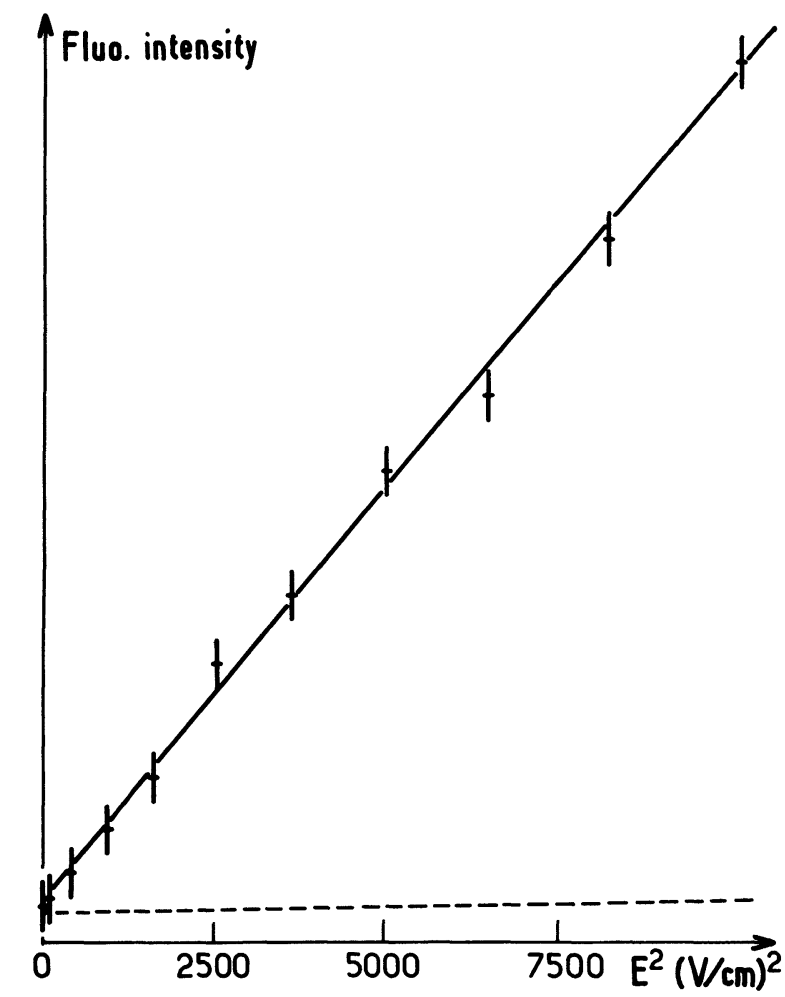

Fig. 5. - Fluorescence intensity versus $E^{2}$ at low field values $(0-100 \mathrm{~V} / \mathrm{cm})$. Experimental points with $\pm 1 \mathrm{rms}$ noise error bar; continuous line : linear fit; dashed line : signal measured off-resonance.

Table I. - Typical experimental conditions corresponding to resonant excitation of the $6 \mathrm{~S}_{1 / 2}, F=4 \rightarrow 8 \mathrm{~S}_{1 / 2} F=3$ and $4 \mathrm{hf}$ components at $411 \mathrm{~nm}$ and detection of fluorescence photons emitted in the $8 \mathrm{~S} \rightarrow 6 \mathrm{P}_{1 / 2}$ decay at $761 \mathrm{~nm}$ (branching ratio $=0.223$ ). The expected yield is computed using the theoretical prediction $\alpha=-306 a_{0}^{3}$.

\author{
Atomic density at $419 \mathrm{~K}$ \\ Length of the interaction region \\ Electric field \\ Incident photon flux (at $\lambda=411 \mathrm{~nm}$ ) \\ Detection efficiency \\ Detected fluorescence flux \\ Observed fluorescence yield \\ Expected fluorescence yield
}

$$
\begin{aligned}
& 1.9 \times 10^{14} \mathrm{at} / \mathrm{cm}^{3} \\
& 10 \mathrm{~mm} \\
& 400 \mathrm{~V} / \mathrm{cm} \\
& 8 \times 10^{13} \text { photons per pulse }(40 \mu \mathrm{J}) \\
& 4 \times 10^{-3} \\
& 1.1 \times 10^{4} \text { photons per pulse } \\
& 3.4 \times 10^{-8} \\
& 3.9 \times 10^{-8}
\end{aligned}
$$


very probably in collision-induced absorption. We have observed nearly identical resonant signals for very different beam sections $\left(0.1,1\right.$ and $\left.50 \mathrm{~mm}^{2}\right)$. This indicates that resonant two-photon photoionization is unlikely in our experimental conditions.

Table I presents a comparison between the observed fluorescence yield (estimated from the photomultiplier gain given by the manufacturer) and the expected fluorescence yield computed from the theoretically predicted polarizability $\alpha$. The agreement is quite satisfactory, in view of the large uncertainty which affects the photomultiplier gain.

\section{Experimental results.}

\subsection{Measuring the ratio $|\alpha / \beta|$.}

4.1.1 Spectra. - By heating up or cooling down the intracavity etalon one can easily sweep the laser frequency over the transition and obtain a spectrum. Between two sweeps the laser polarization is rotated through $\pi / 2$ by rotating a Glan polarizer preceded by a thin half-wave plate. Thanks to the good quality of the selected Glan prism, the possible displacement of the beam affects the fluorescence intensity by an uncertainty estimated $\lesssim 10 \%$. Figure 6 shows this spectrum at $E=500 \mathrm{~V} / \mathrm{cm}$ for polarization parallel or perpendicular to $\mathbf{E}$. In this particular measurement we have used only one channel of the box-car to integrate one photomultiplier output while the other channel was used for the output of the photodiode placed in the laser beam after the cell. Their ratio, which eliminates drifts and fluctuations in the laser intensity, is actually the signal registered in figure 6.

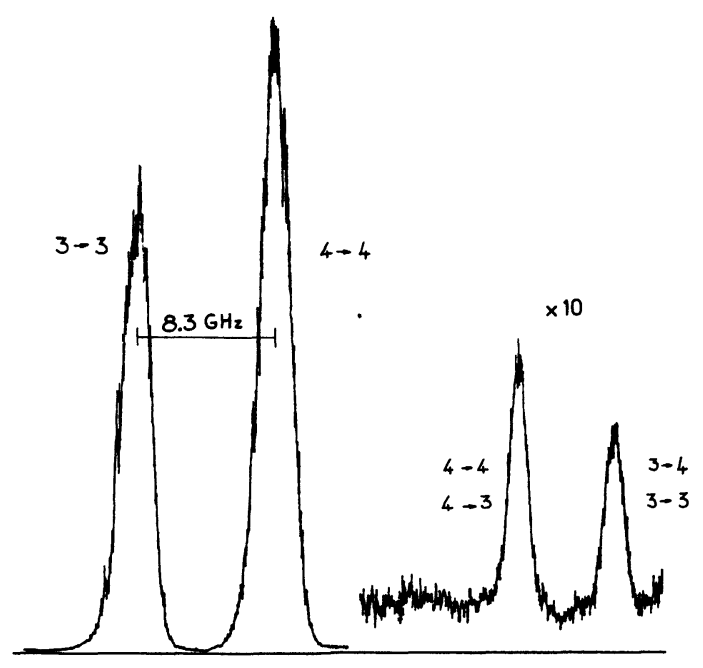

a]

b)

Fig. 6. - Hyperfine spectrum of the Stark-induced $6 \mathrm{~S}_{1 / 2}-8 \mathrm{~S}_{1 / 2}$ transition at $E=500 \mathrm{~V} / \mathrm{cm}$. Fluorescence intensity versus time during approximately linear frequency sweep by thermal drift of intracavity etalon. a) $\varepsilon / / \mathbf{E}$; laser frequency decreases with time; b) $\varepsilon \perp \mathbf{E}$; laser frequency increases with time; $\times 10$ magnification.
(The frequency jitter, not eliminated in this case, turns out to be the major source of noise close to or at resonance.)

Since the $8 \mathrm{~S}_{1 / 2}$ level is not resolved, in perpendicular polarization the four transitions result in two lines. Their heights are functions of the (known) splitting of the $8 \mathrm{~S}_{1 / 2}$ level and of the spectral width of the laser. The separation of the two lines observed in polarization parallel to $\mathbf{E}$ is known to be $8.3 \mathrm{GHz}$. This gives us a scale for measuring the observed width $\omega_{t}$ of the $4-4$ and $3-3$ transitions; we find $\omega_{t}=2.1 \pm 0.3 \mathrm{GHz}$. This width has two contributions $\omega_{\mathrm{t}}^{2}=\omega_{\mathrm{d}}^{2}+\omega_{1}^{2}$, where the Doppler width $\omega_{\mathrm{d}}$ is known $(0.93 \mathrm{GHz})$ and $\omega_{1}$ is the unknown laser width. This yields $\omega_{1}=$ $1.9 \pm 0.3 \mathrm{GHz}$.

The heights of the two lines observed with perpendicular polarization can be computed from formulae (5) and (6) taking into account the two overlapping transitions :

$$
\begin{aligned}
& 4-(3 \& 4): I_{4}^{\perp}=32.0 \beta^{2}, \\
& 3-(3 \& 4): I_{3}^{\perp}=25.7 \beta^{2},
\end{aligned}
$$

leading to the ratio $I_{4}^{\perp} / I_{3}^{\perp}=1.25$.

With parallel polarization the heights of the two lines :

$$
\begin{aligned}
& 4-4: I_{4}^{\|}=36 \alpha^{2}, \\
& 3-3: I_{3}^{\|}=28 \alpha^{2},
\end{aligned}
$$

are expected to be in the ratio $I_{4}^{\|} / I_{3}^{\|}=1.29$.

The observed ratios $I_{4}^{\|} / I_{3}^{\|}=1.40 \pm 0.04$ and $I_{4}^{\perp} / I_{3}^{\perp}=1.38 \pm 0.02$ differ slightly but significantly from the predicted values. However both ratios are affected by roughly the same $8 \%$ discrepancy, and this suggests that the cause is the same. Very likely it is associated with the change in laser frequency necessary to compare $I_{3}$ to $I_{4}$ : this change is carried out in the same way to measure $I_{3}^{\|} / I_{4}^{\|}$and $I_{3}^{\perp} / I_{4}^{\perp}$ and this would generate the same spurious effects (e.g. slight change in beam structure and position). Since no such frequency change is involved in the measurements of $I_{4}^{\|} / I_{4}^{\perp}$ and $I_{3}^{\|} / I_{3}^{\perp}$ we do not believe them to be biased in this way. However this interpretation cannot be guaranteed, so we conservatively add a $10 \%$ systematic uncertainty to our empirical determination of $\left(\alpha^{2} / \beta^{2}\right)$.

Comparing now the observed ratios :

$$
I_{4}^{\|} / I_{4}^{\perp}=25.4 \pm 2.5 ; \quad I_{3}^{\|} / I_{3}^{\perp}=26 \pm 2.5
$$

(uncertainties account for noise in the base line) with the predicted values $1.12 \alpha^{2} / \beta^{2}$ and $1.09 \alpha^{2} / \beta^{2}$, we deduce $\alpha^{2} / \beta^{2}=23 \pm 5$. The uncertainties include both the base line uncertainty and the systematic uncertainties mentioned earlier.

Another source of systematics might be the inhomogeneity of $\mathbf{E}$. Since the intensities of the lines are so much larger for « parallel » polarization, an inhomogeneity which introduces a small component of $\mathbf{E}$ 
transverse to its average direction and to the beam would increase the fluorescence in the "perpendicular» polarization case while leaving it practically unchanged in the "parallel» case. This would artificially decrease $|\alpha / \beta|$.

A numerical estimation has been performed considering the finite size of the electrodes (Fig. 4) and of the laser beam (diameter $<2 \mathrm{~mm}$ ) and assuming the glass cell is at ground potential (the most pessimistic assumption). We obtain the rms tilt of the field in the interaction region :

$$
\left\langle\theta^{2}\right\rangle^{1 / 2} \lesssim 2 \times 10^{-2} \mathrm{rad} .
$$

The correction to $|\alpha / \beta|(\sim 0.4 \%)$ turns out to be negligible. So we obtain $|\alpha / \beta|=4.8 \pm 0.5$.

\subsection{ObSERVIng $P^{(2)}$. Determination OF THE Sign OF $\alpha / \beta$.}

4.2.1 Hanle effect. - Figure 7 shows the dependence of the polarization signal $p_{2}$ (Eq. (14)) on the magnetic field. It is quite consistent with the expected dispersion shape.

From $\alpha / \beta$, we can predict $p_{2}^{*}$. The ratio of the observed to expected value measures the (atomic and optical) depolarization. The Hanle width deduced from the lifetime of the $8 \mathrm{~S}$ state [11] is 5.2 gauss. According to equations (13-15) the expected value of $p_{2}^{*}$ is $7.9 \%$. Since the plate used to polarize the incident beam had a retardation of $(78 \pm 2)^{\circ}$ instead of $90^{\circ}$ the expected maximum is in practice $(6.4 \pm 0.3) \%$.

The observed Hanle width of 7.4 gauss (Fig. 7) and the observed maximum polarization ratio of $4.6 \%$, indicate that the atoms and the fluorescence light are not too much depolarized, so that the measurement of $P^{(1)}$ can reasonably be undertaken in these conditions.

4.2.2 Sign of $\alpha / \beta$. - The other purpose of measuring $P^{(2)}$ is to determine the sign of $\alpha / \beta$ : for a given handi-

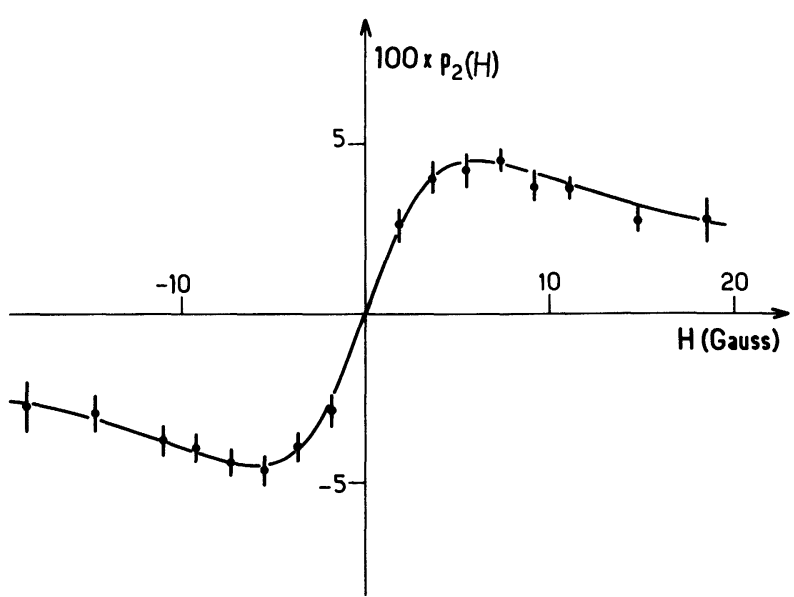

Fig. 7. - Hanle effect detection of the electronic polarization $\mathbf{P}^{(2)}$ created along the laser beam $(F=4 \rightarrow F=4$ excitation) : polarization signal versus magnetic field (experimental points with $\pm 1 \mathrm{rms}$ noise error bar; solid line : least-square fit with a dispersion shape). ness of the trihedral $\left(\xi \hat{\mathbf{k}}, \hat{\mathbf{k}}_{\mathrm{f}}, \hat{\mathbf{H}}\right)$, the sign of $p_{2}$ depends on the sign of $\alpha / \beta$ according to equation (13). In our measurements $(\xi \hat{\mathbf{k}}, \hat{\mathbf{k}} \mathbf{f}, \hat{\mathbf{H}})$ is direct and $p_{2}$ is found to be positive so that $\alpha / \beta$ is negative :

$$
\alpha / \beta=-4.8 \pm 0.5
$$

This value is consistent with the theoretical calculations of reference [8] (see above formula (2)).

\subsection{Measuring $M_{1}$.}

4.3.1 Signal/noise ratio considerations. - The dominant source of noise turns out to be the photon noise of the fluorescence. It results from equation (10) that the noise affecting the polarization ratio is equal (but for a factor $1 / \sqrt{2}$ ) to the relative noise in the fluorescence intensity, and approximately proportional to $E^{-1}$. (The frequency jitter of the laser gives no noise in the polarization ratio.) Since $P^{(2)}$ is independent of $E$ and $P^{(1)}$ is $\propto E^{-1}$, increasing $E$ improves the $\mathrm{S} / \mathrm{N}$ ratio for $P^{(2)}$ while leaving it unchanged for $P^{(1)}$. Yet $E$ should remain moderate enough for $P^{(1)}$ to remain large as compared with possible stray polarizations. We have empirically chosen values around $200 \mathrm{~V} / \mathrm{cm}$ and a caesium pressure of $8 \times 10^{-3}$ torr $\left(150^{\circ} \mathrm{C}\right)$.

Each run consists of 50 program cycles measuring $p_{1}^{*}$, followed by 20 for $p_{2}^{*}$. This corresponds to $40 \mathrm{~min}$, approximately the lifetime of the dye in the laser. In accordance with the noise observed when measuring $p_{2}$, we expect and observe a signal/noise ratio better than unity at the end of each run.

4.3.2 Results. - Fifteen runs have been performed at three different values of the electric field $(100,200$ and $300 \mathrm{~V} / \mathrm{cm}$ ). For each run $E\left\langle P^{(1)}\right\rangle /\left\langle P^{(2)}\right\rangle$ and the associated standard deviation are calculated. A $\chi^{2}$ test checks the consistency between the dispersion of the means and the standard deviations $\left(\chi^{2} / v=9.8 / 14\right)$. Therefore it is justified to combine the results of the different runs.

Figure 8 shows the consistency of the expected and observed variation of $P^{(1)} / P^{(2)}$ as a function of $1 / E$. We obtain finally :

$$
\begin{aligned}
P^{(1)} / P^{(2)}(\xi=+1)= & 8.92 \pm 1.0 \text { (stat.) } \pm \\
& \pm 0.3 \text { (syst.) at } E=1 \mathrm{~V} / \mathrm{cm}
\end{aligned}
$$

and using equation(9) :

$$
M_{1} / \beta=(-9.15 \pm 1.0 \text { (stat.) } \pm 0.3 \text { (syst.) }) \mathrm{V} / \mathrm{cm}
$$

or, combining the uncertainties quadratically :

$$
M_{1} / \beta=(-9.15 \pm 1.05) \mathrm{V} / \mathrm{cm} .
$$

Then with our determination of $\alpha / \beta$, combining again the uncertainties quadratically we obtain :

$$
M_{1} / \alpha=(1.91 \pm 0.30) \mathrm{V} / \mathrm{cm}
$$




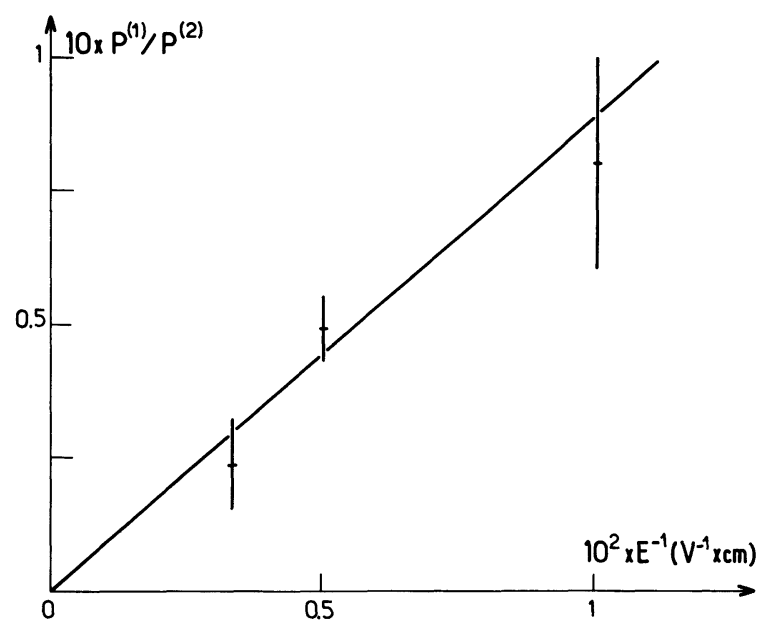

Fig. 8. - Ratio $P^{(1)} / P^{(2)}$ versus inverse of electric field. Data points with $\pm 1 \mathrm{rms}$ noise error bar. Straight line : least-square fit constrained to pass through the origin.

Finally, considering the theoretical value of $\alpha$ given in $\S 1$, the value of $M_{1}$ is :

$$
M_{1}=(-3.11 \pm 0.48) \times 10^{-5}\left|\mu_{\mathrm{B}}\right| / c .
$$

(The quoted uncertainty does not include the theoretical uncertainty of $\alpha$.) This value is in good agreement with the semi-empirical estimate (formula (3)).

\section{Conclusion.}

This experimental work has provided us with the parity-conserving amplitudes characteristic of the highly forbidden $6 \mathrm{~S}_{1 / 2}-8 \mathrm{~S}_{1 / 2}$ transition, namely : $M_{1}$ and Stark-induced amplitudes (the latter associated with the off-diagonal scalar and vector polarizabilities $\alpha$ and $\beta$ ). Within our present accuracy $(10-15 \%)$ the results fully confirm the validity of the atomic model used to predict them.

One might expect the next step to be a measurement of parity violation in this transition. Yet, a clear parity violation in quantitative agreement with the theoretical predictions has already been observed in the $6 \mathrm{~S}-7 \mathrm{~S}$ transition in our group $[2,3]$, then confirmed by other authors [4]. In view of the unusually long integration times in our $6 \mathrm{~S}-7 \mathrm{~S}$ measurements $(5 \mathrm{~h}$ for $\mathbf{S} / \mathbf{N}=1$ ), our first priority is to improve the technique. Detecting stimulated emission rather than fluorescence promises a substantial increase of the detection efficiency [12]. For this first step we prefer the $6 \mathrm{~S}-7 \mathrm{~S}$ transition, more favourable in view of its easier wavelength and of its larger parity-violating amplitude.

But as soon as the statistics have been improved in the $6 \mathrm{~S}-7 \mathrm{~S}$ transition it will immediately become interesting to extend the technique to $6 \mathrm{~S}-8 \mathrm{~S}$. Observing the expected reduction of the parity violating amplitude (by a factor $\sim 2.4[8]$ ) will provide a crucial test of the atomic theory necessary to extract the electroweak parameter from the experimental results.

\section{References}

[1] Bouchiat, M. A. and Bouchiat, C., Phys. Lett. B 48 (1974) $111 ; J$. Physique 35 (1974) 899 and 36 (1975) 493.

[2] Bouchiat, M. A., Guena, J., Hunter, L. and Pottier, L., Phys. Lett. B 117 (1982) 358.

[3] Bouchiat, M. A., Guena, J., Pottier, L. and Hunter, L., Phys. Lett. B 134 (1984) 463.

[4] Gilbert, S. L., Noecker, M. C., Watts, R. N. and Wieman, C. E., Phys. Rev. Lett. 55 (1985) 2680.

[5] Herrmann, P. P., Hoffnagle, J., Schlumpf, N., Telegdi, V. L. and Weis, A., J. Phys. B (to be published).

[6] JACQUIER, Ph., Thèse de 3e cycle (1982), Paris, unpublished.

[7] Bouchiat, M. A., Guena, J. and Pottier, L., J. Physique 46 (1985) 1897.
[8] Bouchiat, C., Piketty, C. A. and Pignon D., Nucl. Phys. B 221 (1983) 68.

[9] Sushkov, O. P., Flambaum, V. V. and Khriplovich, I. B., J.E.T.P. 48 (1978) 37 ; and Phys. Lett. A 67 (1978) 177.

Dzuba, V. A., Flambaum, V. V., Silvestrov, P. G. and Sushrov, O. P., Phys. Scripta 31 (1985) 275.

[10] Hoffnagle, J., Roesch, L. Ph., Telegdi, V. L., Weis, A. and Zehnder, A., Phys. Lett. A 85 (1981) 143;

Bouchiat, M. A., Guena, J. and Pottier, L., J. Physique Lett. 45 (1984) L-61 ;

Gilbert, S. L., Watts, R. N. and Wieman, C. E., Phys. Rev. A 29 (1984) 137.

[11] MareK, J., Phys. Lett. A 60 (1977) 190.

[12] Bouchiat, M. A., JACQuier, Ph., LintZ, M. and Pottier, L., Opt. Commun. 56 (1985) 100. 